\title{
Higgsless Electroweak Symmetry Breaking
}

\section{Christophe Grojean*}

Service de Physique Théorique, CEA Saclay, France

E-mail: grojeanespht.saclay.cea.fr

We present Higgsless models where the electroweak symmetry breaking is achieved by suitable boundary conditions for the gauge fields. We study tree level corrections to precision electroweak physics. Such models inherit from their similarity with technicolor theories a large contribution to the oblique parameters, $S$ in particular. It is possible to suppress $S$ using brane induced kinetic terms and unequal left-right bulk gauge couplings, paying the price of heavy KK modes. In the allowed region, they are eventually ineffective in restoring perturbative unitarity in $W$ scattering above $2 \mathrm{TeV}$. Actually such problem can be easily solved by delocalizing the light fermions in the bulk, which lowers their couplings to the KK gauge bosons. Some tension remains between obtaining a large top quark mass and the correct value of the $Z b \bar{b}$ couplings. Some extra dimensional descriptions of "topcolor" models, where there are two separate strongly interacting sectors contributing to electroweak symmetry breaking, offer some promising directions to alleviate this tension.

International Europhysics Conference on High Energy Physics

July 21st - 27th 2005

Lisboa, Portugal

\footnotetext{
* Speaker.
} 


\section{Higgsless models}

Notwithstanding the amazing success of the Standard Model (SM) in describing high energy physics, we are still missing experimental information about its main ingredient: the mechanism of electroweak symmetry breaking. This lack has left open space for theoretical speculations and for pursuing more or less radical alternatives. The main theoretical motivation is the need to stabilize the Higgs mass against radiative correction. A recent new proposal is the Higgsless scenario [1] (see [2] for an exhaustive list of references extending the original proposal). In extra dimensions, it is indeed possible to break gauge symmetries via boundary conditions, without any light scalar appearing in the theory. Now, the scattering amplitude of longitudinal $W$ bosons is unitarized by the gauge boson resonances, rather than by the Higgs field [3], thanks to the following sum rules relating the masses and the effective couplings of the KK modes:

$$
\begin{aligned}
g_{W W W W}^{2}-e^{2}-\sum_{k} g_{W W Z_{k}}^{2} & =0 ; \\
4 M_{W}^{2} g_{W W W W}^{2}-3 \sum_{k} g_{W W Z_{k}}^{2} M_{Z_{k}^{2}} & =0 .
\end{aligned}
$$

These sum rules are a direct consequence of 5D gauge invariance and they hold even in presence of gauge symmetry breaking boundary conditions.

An enlarged bulk gauge symmetry $S U(2)_{L} \times S U(2)_{R} \times U(1)_{B-L}$ together with a warped background yields a double advantage [4]: a custodial symmetry protects the correct $M_{Z} / M_{W}$ ratio and the warping raises the resonance masses to a realistic level. Similarly, fermion masses can be generated by boundary conditions [5].

Such models also show several similarities with technicolor models via the AdS/CFT correspondence, in particular large oblique corrections are expected. Indeed, in the simplest model $S$ turns out to be of order one, resulting from the tree level mixing with the KK modes. Before discussing the details of precision physics, we will briefly summarize the structure of the model $[1,6]$. We will consider a bulk $S U(2)_{L} \times S U(2)_{R} \times U(1)_{B-L}$ gauge theory on an $\mathrm{AdS}_{5}$ background, working in the conformally flat metric. The AdS curvature $R$ is assumed to be of order $1 / M_{P l}$, however it is a freely adjustable parameter. The parameter $R^{\prime}$ sets the scale of the gauge boson masses, and will therefore be $R^{\prime} \sim 1 / \mathrm{TeV}$. We denote the $5 \mathrm{D}$ gauge couplings by $g_{5 L}, g_{5 R}$ and $\tilde{g}_{5}$. Electroweak symmetry breaking is achieved by the boundary conditions that break $S U(2)_{L} \times S U(2)_{R} \rightarrow S U(2)_{D}$ on the TeV brane and $S U(2)_{R} \times U(1)_{B-L} \rightarrow U(1)_{Y}$ on the Planck brane. We also consider kinetic terms allowed on the branes (on the UV brane: $r$ and $r^{\prime}$ for the $S U(2)_{L}$ and $U(1)_{Y}$ unbroken groups; on the IR brane: $\tau$ and $\tau^{\prime}$ for the $U(1)_{B-L}$ and $S U(2)_{D}$ unbroken groups). One combination of parameters is fixed by the $W$ mass, while the matching of the $4 \mathrm{D}$ couplings $g, g^{\prime}$ determines two more parameters. Therefore one can pick as free parameters of the theory the following set: $R, g_{5 R} / g_{5 L}$, $r, r^{\prime}, \tau, \tau^{\prime}$

\section{Oblique Corrections}

In order to compare Higgsless models to precision electroweak measurements, we need to compute the Peskin-Takeuchi parameters $S, T$ and $U$. We use such parameters to fit the Z-pole observables at LEP1. In [7], Barbieri et al. proposed an enlarged set of parameters, to take into 
account also differential cross section measurements at LEP2. However, the only new information contained by the new parameters is the bound on four-fermi operators generated by the exchange of KK bosons, that we take into account to bound the lighter resonances at LEP2 and Tevatron. Effectively, our $S, T$ and $U$ are linear combinations of the parameters in [7].

In [8] we computed the oblique corrections in the standard way, in terms of mass eigenstates, in the limit where the light fermions are localized on the Planck brane. In the basic model, with $g_{5 L}=$ $g_{5 R}=g_{5}$ and vanishing localized kinetic terms, the leading contribution to $S$ in the $1 / \log R^{\prime} / R \approx .3$ expansion is: $S \approx 6 \pi /\left(g^{2} \log R^{\prime} / R\right) \approx 1.15$ while $T \approx U \approx 0$. This value of $S$ is clearly too large to be compared with the experimental result ${ }^{1}$.

As we already mentioned, however, the theory has more parameters. We first study the effect of asymmetric bulk gauge couplings and Planck brane kinetic terms. The leading contribution to $S$ is now suppressed by a factor $1 /\left(1+g_{5 R}^{2} / g_{5 L}^{2}\right)$ and by a factor $1 /\left(1+r / R / \log R^{\prime} / R\right)$ while, again, $T \approx U \approx 0$. Now, in case of large $g_{5 R} / g_{5 L}$ ratio (or large $S U(2)_{L}$ kinetic term) $S$ is suppressed. However, the $W$ mass squared is also parametrically multiplied by the same factor. This means that the smaller $S$ the larger the scale of the KK resonances, $1 / R^{\prime}$. So, in order to have small corrections we possibly enter a strong coupling regime, where the above calculation becomes meaningless.

Another set of parameters are the TeV kinetic terms. The $S U(2)_{D}$ kinetic term appears at linear order, and effectively multiplies $S$ by a factor $1+\tau / R$. The $U(1)_{B-L}$ kinetic term gives a negative contribution to $S$ at the quadratic order, $-8 \pi\left(g^{2}-g^{\prime 2}\right) \tau^{\prime 2} /\left(g^{2} R \log R^{\prime} / R\right)^{2}$. So, $S$ vanishes for $\tau^{\prime} \approx 0.15 R \log R^{\prime} / R$. However, another effect is to make one of the $Z^{\prime}$ lighter, namely the one that couples with the hypercharge.

We also numerically scanned the parameter space to seek for a region where the model is not ruled out. For different values of $g_{5 R} / g_{5 L}$, we scanned the $\tau-\tau^{\prime}$ space. Requiring both $|S|$ and $|T|$ to be smaller that 0.3 , there is an allowed region only for large ratio, $g_{5 R} / g_{5 L}>2.5$, where the theory is most likely strongly coupled. These results are in agreement with similar studies in [9] and [7].

\section{Reducing $S$ by delocalizing the fermions in the bulk}

We have studied the feasibility of the Higgsless models when facing precision electroweak tests. As originally proposed, the model seems to be disfavoured by the experiments, if one wants strong coupling to arise above $3 \mathrm{TeV}$. However, there is a simple solution that avoids such problems [10], namely to allow the light fermions leaking into the bulk. A simple 5D parameter, $c_{L}$, controls the localization of the fermion along the extra dimension: for $c_{L}>1 / 2$ (resp. $c_{L}<1 / 2$ ) the fermion is localized on the UV brane (resp. IR brane). In the case of almost flat fermions, $c_{L} \approx 1 / 2, S$ vanishes and the resonances almost decouple with the light fermions, see fig. 1 . The direct bounds are then easily avoided and the KK masses can be lowered increasing $R$, thus raising the cut-off of the theory. Therefore, a scenario with $600 \mathrm{GeV}$ resonances and a perturbative regime up to $10 \mathrm{TeV}$ is allowed as estimated by $5 \mathrm{D}$ naive dimensional analysis $\left(\Lambda_{\mathrm{NDA}} \sim \frac{24 \pi^{3}}{g_{5}^{2}} \frac{R}{R^{\prime}}\right)$ corrected

\footnotetext{
${ }^{1}$ Actually, this number should not be compared with the usual SM fit, but we should disentangle the contribution of the Higgs. Namely, it is enough to do the fit assuming a large Higgs mass, equal to the cut-off of the theory [7]. We are also neglecting loop corrections from the gauge KK modes.
} 
by a numerical factor of roughly $1 / 4$ as obtained from an explicit calculation [11] of the scattering amplitude including inelastic channels.
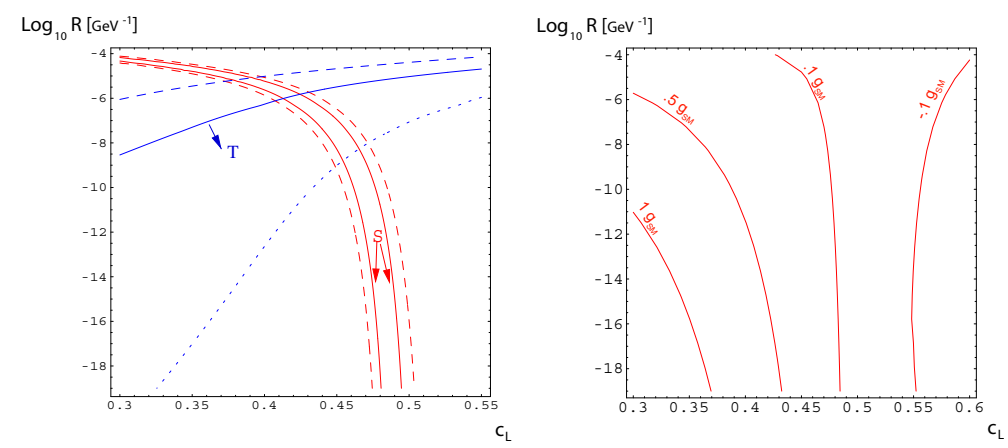

Figure 1: On the left, contours of $S$ (red), for $|S|=0.25$ (solid) and 0.5 (dashed) and $T$ (blue), for $|T|=0.1$ (dotted), 0.3 (solid) and 0.5 (dashed), as function of the UV scale, $R$, and $c_{L}$, the parameter controling the localization of the fermion along the extra dimension. On the right, contours for the generic suppression of fermion couplings to the first resonance with respect to the SM value. In particular we plotted the couplings of a lh down-type massless quark with the $Z^{\prime}$. The region for $c_{L}$, allowed by $S$, is between $0.43 \div 0.5$, where the couplings are suppressed at least by a factor of 10 .

In this simple construction, some tension remains between obtaining a large top quark mass and the correct value of the $Z b \bar{b}$ couplings, which can be alleviated in some extra dimensional descriptions of "topcolor" models, where there are two separate strongly interacting sectors contributing to electroweak symmetry breaking [2].

\section{Acknowledgments}

I would like to thank Csaba Csáki for his invitation to present this work. I am supported in part by the RTN European Program MRTN-CT-2004-503369 and by the ACI Jeunes Chercheurs 2068.

\section{References}

[1] C. Csáki, C. Grojean, L. Pilo and J. Terning, Phys. Rev. Lett. 92, 101802 (2004).

[2] G. Cacciapaglia, C. Csáki, C. Grojean, M. Reece and J. Terning, Phys. Rev. D to appear, hep-ph/0505001.

[3] C. Csáki, C. Grojean, H. Murayama, L. Pilo and J. Terning, Phys. Rev. D 69, 055006 (2004).

[4] K. Agashe, A. Delgado, M. J. May and R. Sundrum, JHEP 0308, 050 (2003).

[5] C. Csáki, C. Grojean, J. Hubisz, Y. Shirman and J. Terning, Phys. Rev. D 70, 015012 (2004).

[6] Y. Nomura, JHEP 0311, 050 (2003).

[7] R. Barbieri, A. Pomarol, R. Rattazzi and A. Strumia, Nucl. Phys. B 703, 127 (2004).

[8] G. Cacciapaglia, C. Csáki, C. Grojean and J. Terning, Phys. Rev. D 70, 075014 (2004).

[9] J. L. Hewett, B. Lillie and T. G. Rizzo, JHEP 0410, 014 (2004).

[10] G. Cacciapaglia, C. Csáki, C. Grojean and J. Terning, Phys. Rev. D 71, 035015 (2005).

[11] M. Papucci, hep-ph/0408058. 